\title{
Data Driven Method of Iron and Steel Enterprise Cost Management System Research
}

\author{
Daqing ZHANG \\ Department of Business Management Dalian University of Technology, Dalian, PR China \\ Jilin Academy of Agricultural Science and Technology, Jilin, PR China \\ Email:dzhang@vip.163.com,tel:135-9626-2279 \\ Xiaobing LIU \\ Department of Business Management Dalian University of Technology, Dalian, PR China
}

\begin{abstract}
The modern cost management emphasizes the strategic, targeted, this requires a higher level of cost management method, need to be more focused on the operation cost of the whole system. Therefore, a real-time response enterprise production situation and real-time control of production cost management method is the modern iron and steel enterprises are in urgent need of management methods. In this only is given priority to with learning method of data driven systems, discusses the data driven approach in the application of the iron and steel enterprise cost management. The modern cost management emphasizes the strategic, targeted, this requires a higher level of cost management method, need to be more focused on the operation cost of the whole system. Therefore, a real-time response enterprise production situation and realtime control of production cost management method is the modern iron and steel enterprises are in urgent need of management methods. In this only is given priority to with learning method of data driven systems, discusses the data driven approach in the application of the iron and steel enterprise cost management.
\end{abstract}

KEYWORD: Data driven; Cost management; Iron and steel enterprises

\section{INTRODUCTION}

At present, the iron and steel enterprise cost management is a prominent problem facing the accumulation of a large amount of data and lack of effective means of data processing and cost management method for cost plan, cost forecast, cost decision-making, provide scientific guarantee. Processes for the production of iron and steel enterprises has become increasingly complex, more and more product type, product type can be as high as hundreds of special steel manufacturing enterprises, according to the analysis of the cost, processing, faced with huge data, people need to consider a new method to solve complex manufacturing process cost control and management problems.

Data driven approach in solving the practical problems in the process of industrial production has been widely used, in the application of cost management is still rare, mainly in the process of application of data driven method is to use data analysis and mining, and other methods. In this paper, on the basis of Xiao-bing Liu professor team's early work, combined with the thinking of data driven method, discusses design of steel enterprise cost management system method.

\section{DATA ANALYSIS}

Data driven method of cost management is mainly based on the data for the cost decision-making and provide feasible scheme for the standard cost, iron and steel enterprises belong to the manufacturing, the data can be divided into real-time data can also be called an online data, and offline data, namely the historical data. Used in the process of iron and steel enterprise cost accounting is based on the historical data, happened in history happened in the process of iron and steel smelting, processing of data distribution, accounting. In order to improve the timeliness of cost management in iron and steel enterprises, in the real-time data in the process of cost management must be fully utilized. Iron and steel enterprises in the process of manufacturing processes to produce a large number of real-time data, using the scientific method tries to use the realtime data, will make iron and steel enterprise cost management more timely, accurate and efficient.

Data driven steel enterprise cost management methods, mainly used the real-time data in the manufacturing process of iron and steel, unable to meet the requirements in real-time data application history data analysis and processing can enhance data driven approach to a certain extent. The difference between real-time data and historical data 
is compared with the real time data, history data response is the process of production and processing history, and can't get the production processing of new information.

Historical data of the first is characterized by its completeness, historical data is long time accumulation of iron and steel enterprises, the data of the sample space should be a continuous reaction throughout the overall state of space. Historical data of the second characteristic is its integrity, historical data is the data in the process of enterprise production and processing from the data accumulated over time, the data on the timeline is a continuous and complete. Real-time data contrary to historical data, the use of real-time data system response of a process or event properties more minutes. Due to the real-time data only consists of part of the sample, so it often is local information and data loss, data measurement error.

Obviously, historical data contains more than real-time data system information, historical data is the production of the whole process of accumulation of data, and real-time data must be a process of data presented in a timely manner. Therefore, more researchers to explore the use of historical data and analysis, with historical data on the processing method of method than the method of real-time data processing. In the field of data mining, machine learning and intelligent computing theory and methods are based on historical data, we think that has been proved by practice as much as possible in history data processing method to deal with real-time data.

That using the method of processing history data processing real-time data, there should have two conditions, the first, exists in the process of cost management requirements, long-term and short-term for long-term cost decision involves a longer time span, need to use the data to do as much as possible; And short-term job such as cost plan, cost control, requires real-time data as a basis for adjustment to the method of management. Second, at present, the development of computer technology, can realize fast computation, makes many offline algorithm can be transplanted into the real-time data processing.

In fact, it can be expected that the future more complex data processing method can easily be incorporated into the real-time processing. Later, the boundaries of offline and online data will be blurred, offline data will be more complete or partial support or enhance the online task.

\section{MODELING}

Data driven approach is not conflict with the method of model, in fact, a data-driven method can also be based on the model. Data driven model can be divided into direct and indirect two kinds, direct data driven models such as neural network model, in the stage of formation of model using the historical data, once the model was established that can use it to deal with the real-time data. Indirect data driven model mainly from data to extract features, when dealing with real-time data such as classifying the data preprocessing in advance.

Application in the process of iron and steel enterprise cost management, using historical data modeling, the research focuses on the definition of cost difference analysis and cost responsibility. Because the data is a process of information, before with the standard of cost plan, cost will produce differences, gap analysis is particularly important. Because modern steel enterprise has risen thousands of work nodes, processing time on the analysis of many nodes one by one is too difficult, we need in iron and steel enterprise cost management designing selection model, applications such as data mining tool to filter, filter production and processing of key nodes or problems more easily work nodes, these nodes are focused on analysis of cost difference reasons, management control planning is put forward.

This management mode can't do the real-time control, if you can use to cost control, real-time data will greatly reduce the cost difference exists, will make iron and steel enterprise cost management more accurate and scientific. Figure 1 shows a combination of real-time cost management model framework, in the long run can use historical data to find out the key to cost management data mining process, in the short term can be used to real-time data, to control the cost difference in real-time data model into the historical data model found in the law and the key work nodes, make real time control more targeted. [1]

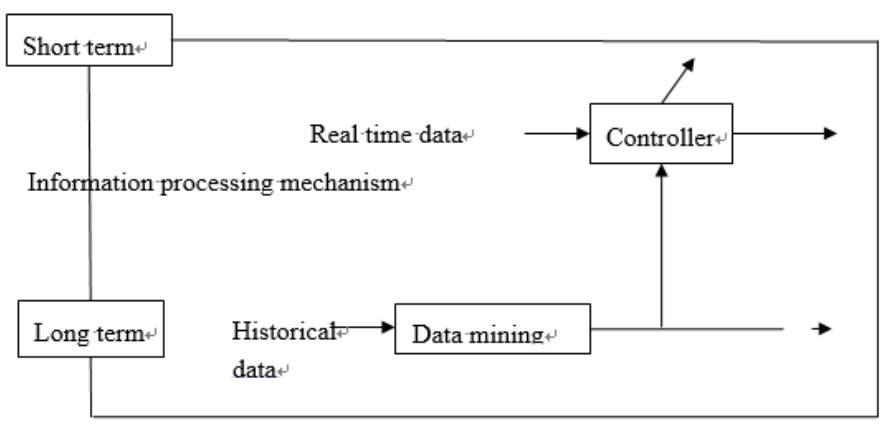

Figure 1. Integrated cost management model.

To deal with the problem of iron and steel enterprise cost management, data driven model will be used in real-time data processing, which is realtime monitoring the cost of their unit of work on iron and steel enterprise, once appear, deviates from the standard cost, warning and processed in a timely manner. Because of the complexity of the iron and steel enterprise production, the resulting costs a lot of information in real time, relying on data driven 
model alone is still difficult to complete in time. Of cost accounting, distribution model, although it is historical data model, but in the role of iron and steel enterprise cost management still exists, data-driven model can be used as the iron and steel enterprise cost management an important supplement.

Production of iron and steel enterprises is a kind of large-scale complex production operation, aiming at its cost management model of a production unit is easy to build, but for the cost management of the whole machining system modeling becomes very difficult. Iron and steel enterprise cost management system belongs to the hierarchical structure, low level of cost accounting, evaluation, data statistics, and analysis is easy to do, is easy to establish related model. But for high cost forecast, cost decisionmaking work, which involves many global variable constraint condition is more complex, involving dispatching layer or design task scheduling and optimization and decision-making layer. In this case, the data driven model can perform its function, use a lot of offline data can establish a stable data driven model.

\section{APPLICATION}

Iron and steel enterprise cost management system level institutions as shown in figure2, which listed in the concept of the iron and steel enterprise cost management system. [2]

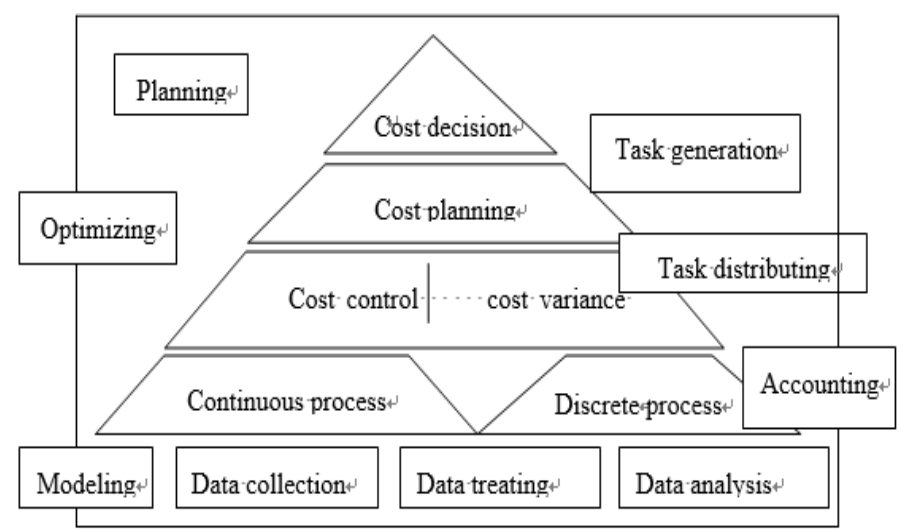

Figure 2. Iron and steel enterprise cost management system structure.

The first level is now doing better in iron and steel enterprise, cost accounting measurement, the continuous and batch processes involved in production and processing of iron and steel enterprise, in the process involves the cost data of statistical work. Data collection, data processing and data analysis are at this level, this level is also the most improve the level of the whole system to collect data, this level contact, of course, the most should be the historical data.

The second level is the iron and steel enterprise cost control and cost variance analysis layer, iron and steel enterprises in this level of cost information check out work, the actual cost information and standard data, search cost differences, analyzes the reasons of differences formation. It involves iron and steel enterprise production real-time data, the control and variance analysis is the main application of datadriven methods.

The third level is the iron and steel enterprise cost plan layer, in this level involves the process of cost plan, cost standards, this level is associated with the cost of top executives, conducted on the basis of cost analysis, optimization of the formulation of the cost of plan and standard cost. The application of this layer is mixed model, namely the model-driven method and a mixture of data-driven methods.

Figure 3 shows the standard cost in the iron and steel enterprise cost management system for the establishment of process model, here include the formulation of standard cost calculation, standard cost control and cost assessment, etc. The whole process is a data drive and the mixture of model driven, the operation standard cost calculation is based on historical data of model driven method, according to the existing processing iron and steel enterprise operation model for the calculation of standard cost. The calculation of standard cost variances is to use the method of data mining to discover the formation of the iron and steel enterprise cost difference characteristics and attributes, and then select the key differences between production and management. In the process of cost difference analysis takes advantage of the real-time data and historical data, adopted the approach of data driven analysis of cost difference formation reasons and control.

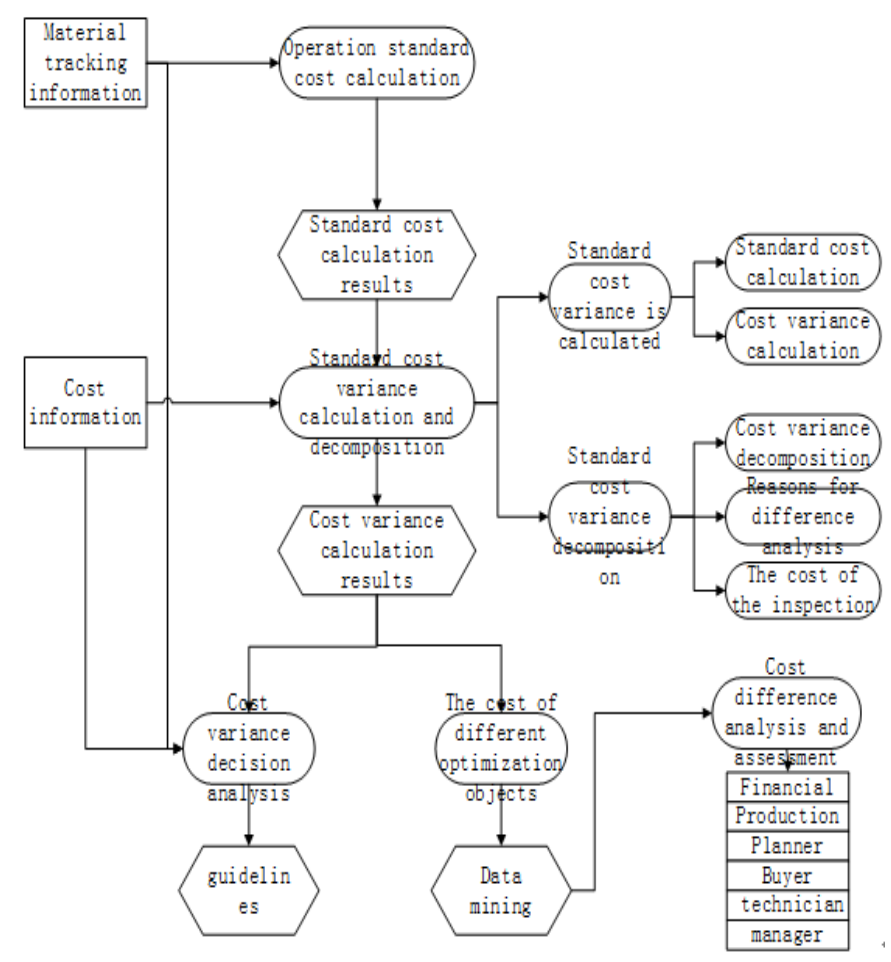

Figure 3. Iron and steel enterprise standard cost management process model. 


\section{CONCLUSIONS}

Data-driven method in the application of iron and steel enterprise cost management system, may say is not what the new method. But before applications are often subject to the conditions of data timeliness, such as data collect, data storage, data error and so on. With the advent of the era of big data, of computer aided technology is used in great quantities in the industrial process including CAPP, CAD/CAE, PDM, MES and other technologies, have been implemented for data collection and management in the whole process of the industrial process. A lot of offline data support makes a datadriven method and theory of management by the wider attention, data driven method of system in the new era of big data still faces many challenges and opportunities. For high-level management activities to develop data-driven method, establishment of intelligent decision support system is a developing direction of management of large-scale industrial processes.

\section{REFERENCES}

[1] HOU Zhong-Sheng; WANG Zhou. 2013. From modelbased control to data-driven control: Survey, classification and perspective. Information Sciences, 235(2013), 3-35.

[2] XU Jian-Xin; HOU Zhong-Sheng. 2009. Notes on Datadriven System Approaches. ACTA AUTOMATICA SINICA2009.6, 669-674. 